\title{
El tránsito a la vida adulta de los jóvenes atendidos desde los servicios sociales ${ }^{1}$ The path of adulthood for youth in social service care
}

\author{
Martin Goyette \\ UNIVERSIDAD DE QUEBEC
}

\section{Resumen}

Los jóvenes que fueron acogidos en medios sustitutivos generalmente encuentran bloqueos importantes en sus transiciones diversas a la vida adulta. En comparación con otros jóvenes, acumulan más dificultades psicosociales y rupturas con el mundo familiar. Paradójicamente, están confrontados más a menudo con la exigencia de autonomía y de independencia.

Consciente de los problemas que plantea el apoyo a los jóvenes a la hora de finalizar su estancia en los centros de juventud cuando se aproximan a la edad adulta, la Asociación de Centros de Juventud de Quebec implantó el Proyecto de Cualificación de Jóvenes (PQJ) en cuatro de estos centros de juventud de Quebec².

El proyecto pretende preparar mejor a estos jóvenes para la vida autónoma, favorecer su cualificación y protegerlos mejor después de su salida. Según los promotores del proyecto, los jóvenes atendidos desde el PQJ constituyen el 10\% de los jóvenes más vulnerables de la clientela de los centros de juventud.

Nuestra pretensión, a partir de este artículo, es precisar los aprendizajes para el desarrollo de la intervención que pueden ser obtenidos desde el PQJ. A partir de la evaluación de este proyecto piloto, podremos presentar su modelo de intervención, particularmente en lo que se refiere a sus componentes distintivos de una intervención más usual. Presentaremos luego de qué manera la intervención contribuye a la autonomía, cualificación y apoyo relacional. En conclusión, pondremos en evidencia los debates que provoca este proyecto, particularmente desde el punto de vista de su generalización en el conjunto de los centros de juventud de Quebec.

PALABRAS ClaVe: tránsito a la vida adulta, evaluación, jóvenes protegidos, intervención, Quebec.

\section{Summary}

Youth who have been in social services care often face important obstacles on their path to adulthood. While they're more prone to psychosocial difficulties and to family discord, Society demands they quickly become autonomous and independent.

In order to better prepare youth for their end of care, the Projet Qualification des Jeunes (PQJ) has been implemented in four youth centers in Quebec. Mindful of the specific issues they face, the project's goal is to better prepare youth for autonomous living, to increase their employability and to better assist them after they leave care. According to the project's promoters, the targeted population represents $10 \%$ of youth centers' most vulnerable population. 
In this article, we seek to present the lessons learned regarding intervention and its development. First, in light of the project's evaluation, we'll present its intervention model, and distinctive elements. Secondly, we'll show how this intervention promotes autonomy, employability and relational support. In conclusion, we'll discuss the challenges it poses, particularly those involving its deployment to all specialized social services in Quebec.

KEY WORDS: path to adulthood, evaluation, youth in social services care, intervention, Quebec.

\section{Difíciles transiciones}

La modificación de las condiciones en la entrada a la vida adulta, observada desde hace algunos decenios, está asociada a la prolongación general de la juventud y a una desincronización de los umbrales de transición a la vida adulta (Bidart, 2006). La inserción de los jóvenes ya no se realiza según un modelo social prácticamente unificado, sino que deja lugar a una cierta individualización de los recorridos, compuesto de avances y retrocesos en las diversas transiciones profesionales, residenciales y familiares (Goyette, 2007 b). Esa prolongación conduce a que esta juventud sea menos independiente o autosuficiente comparada con la juventud de las generaciones anteriores, a causa de que los jóvenes viven más tiempo en casa de sus padres y de que éstos contribuyen a su subsistencia por un tiempo más largo, particularmente durante el período en que los jóvenes estudian (Maunaye et Molgat, 2003).

Ahora bien, si la inserción de los jóvenes en general es complicada por diversas consideraciones, esta dinámica o estos imperativos, asociados con la transición a la vida adulta, constituyen un dilema importante para los jóvenes que tuvieron dificul- tades, particularmente los que entran en la vida adulta después de un paso más o menos prolongado en un recurso de acogimiento familiar (Goyette, 2007a).

La literatura científica refleja con claridad las importantes dificultades para integrarse de esta población. En general, son pocos los que acaban sus estudios secundarios. Entre el $19,2 \%$ y el $43,2 \%$ abandonaron su titulación, de acuerdo con los últimos estudios internacionales censados (Dumaret et al., 1997; Jahnukainen, 2007; Lanctôt, 2006).

En Canadá, consideramos que alrededor de la mitad (entre el 31\% y el 54\%) de ellos trabajan en los primeros meses que siguen a la finalización de su estancia en recursos del sistema de protección de menores (Lanctôt, 2006; Rutman et al., 2006; Pauzé et al., 2004) y lo más frecuente es que los empleos ocupados sean poco remunerados y gratificantes (Goyette et al., 2007; Pauzé et al., 2004).

Algunos confiesan que su salario no es suficiente para colmar sus necesidades; por otra parte, la tercera parte de ellos vive bajo el umbral de la pobreza (Pecora et al., 2006). También sabemos que los jóvenes adultos con antecedentes de acogimiento familiar recurren con más frecuencia a los servicios y ayudas sociales que la población general (Goyette et al., 2006; Tweddle, 2007). Además, los primeros años del retorno a la comunidad, a menudo se caracterizan por la inestabilidad en el ámbito residencial; alrededor de la cuarta parte de los jóvenes experimentarían, por lo menos, un episodio de itinerancia después de llegar a la mayoría de edad, con posterioridad a la salida (Daining \& DePanfilis, 2007; Frechon, 2005; Rutman et al., 2006). Por otra parte, estos jóvenes presentan un cúmulo de problemáticas sociales persistentes (Pauzé et al., 2004) y están representados entre las poblaciones marginales adultas (Tweddle, 2007). 
Este esquema de dificultades muestra que las transiciones a la vida adulta de los jóvenes que salen inmediatamente después de una estancia en los centros de juventud son más arduas que las de los jóvenes pertenecientes a la población en general (Yergeau et al., 2007). Para ciertos jóvenes acogidos, dejar definitivamente este ambiente también quiere decir dejar "las únicas familias y los hogares que tuvieron" (Goyette, 2006).

Ahora bien, aunque esté ampliamente reconocido que los jóvenes acogidos en medio sustituto encuentran dificultades de inserción en Quebec y Canadá, pocos organismos han intentado aceptar el desafío de una intervención constante referida a su inserción socio profesional, y pocas investigaciones contemplaron la transformación de los jóvenes acogidos en familias (Goyette, 2007a; Reid, 2007).

Así, en Quebec desde 2000, varios informes ministeriales, grupos de estudio o de trabajo y opinión subrayan la importancia de tener en cuenta, en la planificación de la intervención, las diferentes problemáticas vinculadas a las transiciones a la vida adulta de los jóvenes que han recibido servicios de los centros de juventud (Goyette et Turcotte, 2004).

Es en este ámbito de influencia en el que el Proyecto de Cualificación de Jóvenes ha sido desarrollado.

\section{Descripción del Proyecto Piloto de Cua- lificación de Jóvenes (PQJ)}

El Proyecto Piloto de Cualificación de Jóvenes fue una iniciativa de la Asociación de Centros de Juventud de Quebec, siendo la continuación de la cumbre de Quebec y la juventud, que colocaba la cualificación de los jóvenes en el centro de un vasto conjunto de medidas para ayudar específicamente a jóvenes en dificultad. El proyecto de la Asociación fue entonces coordinado con una investigación-acción iniciada bajo la denominación de Solidaridad Juventud, en calidad de proyecto "satélite", con el fin de estudiar sus efectos y permitir desarrollar nuevas estrategias de inserción social y profesional para los jóvenes de 16 a 18 años que reciben servicios de los centros de juventud.

En principio, el proyecto ha sido implantado en cuatro regiones de Quebec, a saber: Abitibi-Témiscamingue, Laval, Montreal (Centros de juventud y familia Batshaw) y Outaouais, reuniendo a ochenta jóvenes, es decir, veinte por centro de juventud.

Con el fin de prevenir la marginación de los jóvenes de los centros de juventud, en el momento en que alcanzan su mayoría de edad y cesa su acogimiento, recordemos que el objetivo del programa es aumentar sus capacidades para integrarse socialmente de manera autónoma y de implicarse en el plano profesional.

El PQJ contiene tres aspectos que se refieren cada uno a un objetivo específico:

- Desarrollo de una red social y de apoyo.

- Preparación a la vida autónoma.

- Cualificación con miras a la inserción socio profesional.

El aspecto relativo al desarrollo de una red social y de apoyo vela por que el joven mismo pueda arraigarse a su medio de vida, con la ayuda del profesional interviniente, con el fin de acceder a los recursos que pudiera necesitar. El objetivo es desarrollar redes de apoyo y ayuda con las que los jóvenes originarios de centros de juventud puedan contar.

El aspecto relativo a la preparación para la vida autónoma inicia un proceso que permite a los jóvenes asumir sus responsabilidades cuando alcancen su mayoría de edad. El objetivo aquí es el de favorecer los aprendizajes que permitan acceder a una autonomía funcional, sabiendo personalmente buscar y mantener una vivienda, administrar sus finanzas personales, desa- 
rrollar habilidades sociales y preocuparse por sus cuidados personales.

El aspecto relativo a la cualificación con vista a la inserción socio profesional permite la adquisición de conocimientos (saber), técnicas particulares (destreza) y actitudes (saber - ser) esenciales para acceder al mercado del trabajo e integrarse en el cuerpo social. El objetivo aquí es el de integrar por lo menos al 75\% de los jóvenes del PQJ en una trayectoria que lleve a un empleo o a una formación, que permita cualificarse con vista a una ocupación profesional.

\subsection{La clientela del $P Q J$}

El PQJ reunió a 80 participantes que provenían de cuatro regiones de Quebec, 33 chicas y 47 chicos. Los jóvenes reclutados para participar en el proyecto debían tener 16 años en el momento de su adhesión. El reclutamiento de estos jóvenes fue realizado por ocho educadores y la coordinadora ${ }^{4}$, con la referencia de los equipos habituales del centro de juventud. El perfil tipo buscado entre los jóvenes se corresponde con los principales factores de riesgo asociados a una trayectoria de vulnerabilidad. Han conocido en el curso de su vida una historia importante de acogimiento, y probablemente no les será ofrecida la posibilidad de poder reintegrarse al medio familiar; ellos mismos no formularon un proyecto preciso en relación con su escolarización o en relación con una formación profesional al finalizar su estancia en centros de juventud. Manifiestan inseguridad, tienen una baja autoestima, una red social débil, experimentan dificultades en espera de gratificación, y también en respetar las consignas y en ponerse límites.

El PQJ procura aumentar, de modo significativo, las posibilidades de éxito de estos jóvenes con el fin de que se inscriban en un proyecto de vida adulta que les ayude a desarrollarse. La población elegida es, de acuerdo con todo esto, aquella para la que los servicios habituales no son suficientes, y no ha sido adoptado ningún criterio de exclusión.

Además, el acompañamiento a los jóvenes se diferencia de los servicios habituales ofrecidos en centros de juventud en que continúa más allá de la mayoría de edad del joven. Según la edad del joven en el momento de la selección, el control puede continuar hasta los 19 años. Así, es posible estar presente en el momento de la transición a la vida adulta de los participantes, permitiéndoles ser acompañados en su nueva vida de adulto que les hace enfrentarse a nuevas responsabilidades de las que, con mucha frecuencia, no habían sido conscientes. El proyecto se caracteriza también por la intensidad de la intervención, ya que cada profesional interviniente del PQJ trabaja con un máximo de diez jóvenes.

\section{La evaluación del PQJ}

La presentación de los resultados de la evaluación permitirá destacar ciertos posicionamientos en este campo de intervención. La evaluación se apoyó en una metodología fundamentalmente longitudinal mixta, cuantitativa y cualitativa, a fin de desarrollar una adecuada comprensión de las adaptaciones necesarias para la implantación del PQJ (Goyette et al., 2007).

\subsection{Consideraciones metodológicas}

La encuesta entre los jóvenes del PQJ pretende seguir la evolución de la situación de los jóvenes en el plano de su autonomía y cualificación profesional, y documentar la contribución del PQJ a los cambios observados.

El equipo de investigación se apoyó primeramente en los datos recopilados por los profesionales intervinientes del PQJ, a través de los siguientes instrumentos. 
1. ACLSA (Ansell-Casey Life Skills Assessments), que mide la autonomía funcional de los jóvenes, ha sido administrado cada seis meses desde la admisión del joven al PQJ. ACLSA (Versión 3) fue traducido al francés por la ACJQ. Comprende las dimensiones siguientes: tareas de la vida cotidiana (11 enunciados); vivienda y recursos comunitarios (10 enunciados); gestión financiera (8 enunciados); capacidad de cuidar de sí mismo, cuidados de la salud (1 1 enunciados); desarrollo social (19 enunciados); hábitos escolares y de trabajo (19 enunciados).

\section{Una escala de evaluación de severidad} del perfil y una escala de evaluación sobre las disposiciones personales. Con el fin de dotarse de una panorámica sobre la evolución de los jóvenes participantes en el PQJ, los profesionales intervinientes desarrollaron un procedimiento que pretendía establecer las diferentes características de severidad y las disposiciones personales de los jóvenes en diferentes momentos. La escala de severidad del perfil cuenta con nueve dimensiones y la escala sobre las disposiciones personales cuenta con doce. Para la escala de severidad, las dimensiones son: historia de acogimientos; escolaridad; proyecto de futuro (cualificación: formación/ empleo); proyecto de vida (autonomía, vida independiente); presencia de una red social (relaciones significativas); discapacidad intelectual (límites); delincuencia; toxicomanía; salud mental (ansiedad, depresión y otros problemas de salud mental). La tabla de evaluación de la severidad ha sido completado cuatro veces, a la llegada del joven al programa y después, a los seis meses, durante dos años después de la entrada al PQJ. Para la escala de las disposiciones personales, las dimensiones son: inteligencia/talento específico; imaginación/capacidad de simbolizar; vida espiritual/fe; sentido del humor; sentimiento de arraigo; capacidad de proyectarse en el tiempo (en general); carisma; apariencia agradable; curiosidad / necesidad de saber, de comprender; experimentación del éxito; capacidad de percibir lo que suscita en otros; capacidad de "rebotar" (resiliencia). No obstante, debido a la ausencia de datos, la dimensión vida espiritual/fe fue retirada de la escala. La escala de evaluación de las disposiciones personales ha sido completado en los mismos momentos que la prueba precedente, salvo para el segundo momento. La consistencia interna de escalas es satisfactoria y válida ${ }^{5}$.

Por otro lado, para los ochenta jóvenes del PQJ, se realizó una entrevista de investigación semi-directiva con el profesional interviniente del PQJ en otoño de 2004, y otra entre los seis y los ocho meses después. El equipo de investigación administró también sus propios instrumentos de investigación a 61 jóvenes con los que se mantuvo contacto en otoño de 2004, justo antes de que los servicios prestados desde el centro de juventud finalizaran, y después a otras 31 personas, entre los seis y los ocho meses siguientes al final de los servicios prestados.

\section{El calendario biográfico es un instru-} mento que permite recopilar informaciones sobre la trayectoria familiar, la trayectoria residencial, la historia de protección, la trayectoria escolar y la trayectoria profesional, desde el nacimiento del joven.

4. El cuestionario sobre redes sociales permite la reconstitución de las redes sociales y redes de apoyo a partir de generadores de nombres (Bidart et Charbonneau, 2007).

5. Las entrevistas del tipo "relato de vida" permiten que el joven "se cuente" a sí mismo, a partir de un relato retrospectivo. El procedimiento durante estas entrevistas pretende documentar la evolución de la situación del joven a través de varias esferas de su vida, de las relaciones sociales que mantiene así como del sentido que atri- 
buye a sus relaciones sociales en el marco de las transiciones que vive o que vivió.

Las conclusiones de los análisis efectuados sobre los resultados de las escalas de severidad del perfil y de las disposiciones personales muestran que los datos sobre los 19 jóvenes que no fueron entrevistados en otoño de 2004 no eran significativamente diferentes a los obtenidos de los otros jóvenes a su entrada en el programa: ambos grupos obtienen, en T1, resultados promedio similares a ambas escalas, respectivamente un resultado promedio de severidad de 10,8 para los jóvenes que no fueron entrevistados y de 9,8 para los que sí lo fueron. El resultado promedio, medido por la escala de las disposiciones personales, es de 9 para el grupo de jóvenes no entrevistados y de 8 para los otros. Además, un test promedio mostró que las diferencias observadas entre los resultados promedios de ambos grupos no eran estadísticamente significativas. El mismo proceso ha sido efectuado con el fin de asegurarse que los 30 jóvenes que no fueron entrevistados en $\mathrm{T} 7$, sino que lo fueron en T6, no difieren tampoco de los otros. Revela que a su entrada al programa PQJ, los resultados promedios de severidad del perfil y de las disposiciones personales son similares entre los jóvenes que fueron entrevistados una vez, dos veces, o ninguna.

Aunque solamente 61 jóvenes fueron entrevistados por lo menos una vez, para 75 de 80 jóvenes se ha recogido una cantidad de datos suficientes con los profesionales intervinientes, relativos a las intervenciones llevadas por el PQJ y sus repercusiones, así como sobre la inserción de los jóvenes en su tránsito a la vida adulta.

\subsection{Los resultados}

Ya mostramos en otro lugar que, de manera general, (Goyette et al., 2007) la autono- mía de los jóvenes participantes al PQJ mejoró de forma considerable durante la intervención y a partir de ella (medido por el Ansell-Casey Life Skils Assessments, ACLSA). Añadidos a los resultados obtenidos de los análisis sobre los perfiles de severidad y de disposiciones personales, estos resultados muestran claramente que hay una mejora en el perfil de los jóvenes en el curso de su paso por el PQJ.

Así, la severidad del perfil está en disminución constante a lo largo de la intervención PQJ (desviación media de 3,3 puntos de mejoramiento entre $\mathrm{T}_{1}$ y $\mathrm{T}_{7}(\rho<0,001$ $\eta=0,423)$, mientras que las disposiciones personales aumentan en el curso del mismo período (diferencia promedio de 7,9 puntos de mejoramiento entre $\mathrm{T}_{1}$ y $\mathrm{T}_{7}$ $(\rho<0,001 \eta=0,620)$.

Habría, en este sentido, que interrogarse sobre qué éxito puede deberse al hecho de que los jóvenes mismos han podido simplemente madurar en el curso de los tres años que duró el proyecto. Sobre este punto, los análisis cualitativos mostraron claramente el importante papel del PQJ como apoyo activo en el tránsito a la vida adulta, así pues nos quedaría comprender el papel que corresponde a los elementos activos de la intervención. En esta perspectiva, analizamos la trayectoria de los jóvenes en función de las tres esferas de intervención identificadas por el PQJ.

A partir de la codificación de las repercusiones de los principales actores de la red del joven (inhibidor, transmisor, neu$\left.\operatorname{tro}^{6}\right)$, de la dinámica relacional mantenida al respecto y de la puesta en funcionamiento en un proyecto a través de todos los ejes de transición y los procesos de inserción, se ha elaborado un retrato global del joven en cada uno de los dos tiempos de medida de la transición.

Estas representaciones ponen en evidencia la dinámica relacional preponderante del joven (dependencia, independen- 
cia o interdependencia) así como el proceso hacia la autonomía en el que el joven se incluye (itinerancia constructiva o itinerancia vulnerabilizante) (Bellot et Goyette, 2006; Goyette et al., 2006b). Sobre la dinámica relacional, hemos mostrado en otro lugar que la relación de los jóvenes con su ambiente, ya sea de dependencia, de independencia o de interdependencia, debe ser considerada en el análisis de la contribución dinámica de las redes a la inserción de los jóvenes. Así, los jóvenes "negocian" ciertos apoyos, bloqueando algunos en un ámbito vital y aceptando otros. (Goyette, 2007b). Así, desde nuestra perspectiva, el término "itinerancia" es utilizado con el fin de poner delante la naturaleza dinámica del proceso, junto a la posibilidad de oscilaciones y de bifurcaciones que no son necesariamente perjudiciales para la inserción. Es decir, "utilizada como figura más bien que como problema, la itinerancia permite captar mejor las transiciones en términos de las ganancias y las pérdidas que viven estos jóvenes" (Bellot et Goyette, 2006: 24).

De manera general, son mayoría los jóvenes que se inscriben en una trayectoria constructiva al final del PQJ. En efecto, el $56 \%$ de los participantes $(n=42)$ se sitúan en ese tipo de trayectoria al final del proyecto, revelando la amplitud del trabajo efectuado por los profesionales intervinientes del proyecto. A la inversa, el $44 \%$ de los jóvenes $(n=33)$ se reencuentran en una trayectoria vulnerabilizante. Comparando, antes de finalizar la estancia en el centro de juventud (seis a ocho meses antes de la terminación del PQJ), el 32\% $(n=22)$ de estos jóvenes estaban en una trayectoria constructiva. Teniendo en cuenta que los jóvenes que participan en el proyecto tienen un historial cargado de múltiples oscilaciones y bifurcaciones, estas tendencias se muestran reveladoras de la importancia de un proyecto como el PQJ y del trabajo que queda por hacer. También revelan que la situación de los jóvenes puede evolucionar bastante rápidamente en el tiempo, de ahí la importancia de prestar atención a la duración tanto desde el punto de vista de la intervención como desde el punto de vista de la investigación.

\section{Eje de la autonomía}

En la perspectiva del PQJ, el trabajo sobre la autonomía remite especialmente a la dimensión residencial y funcional. La relación con la intervención PQJ resulta primordial, ya que muchos jóvenes no poseen los recursos sociales que permitirían otra alternativa que no fuera la vivienda autónoma. El compromiso de los jóvenes constituye una garantía de éxito de su inserción residencial, excepto para las jóvenes que cohabitan con una pareja fuertemente inhibitoria y cuya influencia bloquea a la del profesional interviniente. La relación positiva y constructiva mantenida con la persona con la que los jóvenes cohabitan es esencial, y vice-versa.

Respecto a los jóvenes que viven en casa de sus padres durante nuestra última recogida de información (hacia los 19 años), la dinámica que permite la estabilidad de la vivienda es diferente: la inserción en una trayectoria ascendente o descendente es tributaria de los recursos paternos y de las características personales inhibitorias de los jóvenes (salud mental, consumo de drogas, delincuencia), más bien que la relación con la intervención, frente a la cual la mayoría de estos jóvenes tiene una actitud independiente.

\section{Eje de la cualificación}

En términos de proyecto, la actualización o el funcionamiento en un proyecto académico o cualificante no necesariamente significa compromiso con una trayectoria cons- 
tructiva, aunque se trate de un buen indicador. En el mismo orden de ideas, la ausencia de proyecto no es sinónimo de fracaso. Las características personales de los jóvenes del PQJ, como también las de su ambiente, tienen un impacto importante sobre las trayectorias e influyen sobre las elecciones de los jóvenes en materia de cualificación. Finalmente, la relación mantenida con el profesional interviniente es un factor discriminante de la trayectoria de los jóvenes. Además, la estabilidad residencial parece una condición sine qua non para la conservación del empleo o de los estudios.

Los que ocupan un empleo o los que estudian (o ambas cosas simultáneamente), se insertan más a menudo en una trayectoria constructiva. Estos jóvenes, evolucionando en itinerarios constructivos, mantienen una relación de interdependencia con su profesional interviniente en el conjunto de los tres ejes del proyecto, están comprometidos en el proceso de intervención y tienen relaciones fuertes y positivas en su red, que desempeñan un papel importante en cuanto a su estabilidad residencial y por extensión favorecen la conservación del empleo. Mientras que por el contrario, de manera general, los jóvenes que se mantienen en itinerancia vulnerabilizante tienen una actitud de independencia frente a la intervención en la esfera del empleo, además de mantener una relación de dependencia frente a agentes inhibitorios y de no tener referentes constructivos no institucionales en su red.

La presencia de un referente fuerte en la esfera escolar, para los jóvenes del PQJ que frecuentan la escuela, facilita la inscripción en una trayectoria constructiva. Este actorreferente no es el profesional interviniente del PQJ, a pesar de que la relación entre este último y los jóvenes sea discriminante de la trayectoria, ya que su acción se sitúa principalmente alrededor de la inserción social. Estos jóvenes también tienen pocos inhibi- dores en su vida, sean relacionales o no. Los jóvenes que estudian y trabajan simultáneamente han sido más activos en la gestión de sus relaciones sociales y de sus problemas de salud mental, lo que les permite una mayor implicación en sus proyectos. La aportación de la intervención del PQJ se traduce en este caso en una intervención en cuanto a la relación consigo mismo y con los demás.

Los jóvenes inactivos en la esfera de la cualificación al término del programa presentan, a su entrada al PQJ, un perfil de severidad más sombrío en comparación con los que son activos en el plano de la cualificación. Recaen más a menudo en una trayectoria vulnerabilizante y mantienen una dinámica relacional de independencia a las repercusiones vulnerabilizantes. Este modelo de inserción es privativo de ciertos jóvenes que mantienen actividades asociales y de pequeña delincuencia.

En términos de inserción en el plan de cualificación, la acción del PQJ se revela global, tanto en lo que se refiere a los pasos en la búsqueda de empleo, como en las percepciones y expectativas frente al mundo del trabajo. En consecuencia, los jóvenes del PQJ presentan una mayor predisposición a integrarse en el mercado del trabajo y han desarrollado más experiencias en lo que concierne a esta ámbito.

Desde el punto de vista de la trayectoria en el empleo, los jóvenes con una red social mínimamente funcional y con pocas problemáticas personales, están generalmente inscritos en una trayectoria de cualificación. Pero para estos últimos, la intervención desde el PQJ no necesariamente logra llevarles hacia mejores empleos o favorecer más la conservación de estos, encontrándose el joven enfrentado a los límites de la oferta de empleo para los jóvenes menos formados. En cambio, el PQJ permite a los que probablemente no habrían llegado solos a mantenerse en un proyecto cualificante, particularmente a los jóvenes con 
problemas de salud mental o de deficiencia intelectual. La intervención del PQJ permite a estos jóvenes encontrar una vía adaptada a sus necesidades, motivarlos y sobre todo, hacerles conscientes de sus fuerzas y debilidades y encontrar soluciones adaptadas a sus bloqueos. Así, trabajar la relación consigo mismo y con los demás, combinándolo con pasos concretos en la búsqueda de empleo y en formación para el empleo, parece una perspectiva prometedora hacia la inserción en un proyecto que cualifica.

\section{Eje de la red}

Hemos comprobado que las redes de jóvenes contribuyen a veces a producir diferencias en cuanto al progreso profesional y residencial. Pues bien, las redes de jóvenes participantes están compuestas en su mayor parte por amigos de la misma edad y del mismo sexo, y además son restringidas para la mayoría de ellos. El tamaño de la red de jóvenes investigados no está vinculada a su impacto sobre la trayectoria: una red limitada no es más inhibitoria que una red extensa, y una red extensa no sostiene más que una red más limitada. No es por tanto sobre la relación estadística (la cantidad) sino sobre la dinámica relacional sobre la que hay que trabajar. Finalmente, el sujeto principal de la red de jóvenes es mayoritariamente un elemento de inserción, aunque resulta un elemento inhibidor en casi una tercera parte de los casos. Además, las redes de chicas y las de los chicos no presentan diferencia significativa en términos de composición, extensión e impacto, así como en términos de actor principal y de su impacto. Aunque existen distinciones entre los sexos en el plano de las relaciones, la composición de la red no es diferente según los sexos. Ahora bien, los resultados de la evaluación también muestran cómo es importante interesarse por las dinámicas particulares de las jóvenes y de los jóvenes. En efecto, ciertas chicas parecen tener un perfil relacional más deteriorado que los jóvenes, identificado con la dependencia relacional.

Así, contrariamente a los resultados de Yergeau et al. (2007), el análisis cualitativo de las dinámicas de inserción y de apoyo muestra que el tránsito a la vida adulta es fuertemente sexuado; las vías para "convertirse en adulto" de los jóvenes que dejan los centros de juventud difieren según el sexo.

De acuerdo con Lanctôt (2006), la evaluación del Programa de Cualificación de Jóvenes (PQJ) muestra que más de la mitad de las jóvenes ha tenido un hijo antes de los 19 años (Goyette, 2007a). Si la maternidad adolescente de estas jóvenes mujeres es el ejemplo de una derogación de las normas sociales sobre la edad requerida (Charbonneau, 2003), se distingue también de otras etapas por su carácter definitivo e irreversible. Así, relación amorosa, cohabitación familiar y accesos al empleo testimonian hoy, procesos progresivos tipo yo-yo y reversibles. La maternidad influye además el curso de otras transiciones. Para varias de estas jóvenes, si la maternidad adolescente parece una oportunidad de liberación y reconocimiento social (Goyette et al., 2006; Manseau, 1997), esto conduce a veces a situarlas en una dinámica relacional basada en una distribución tradicional de roles sociales, donde el hombre es el proveedor, y también en dinámicas relacionales cerradas.

De manera más global, una red que da soporte lleva casi invariablemente a los jóvenes a una trayectoria de inserción constructiva, mientras que una red inhibitoria los empuja principalmente hacia la itinerancia vulnerabilizante. Es posible de establecer tres constataciones principales que conciernen a las modificaciones aportadas en las redes sociales de los jóvenes del PQJ y su trayectoria. En primer lugar, todos los jóvenes cuya red se mejoró se introdujeron en trayectorias constructivas al final de la intervención; en segundo lugar, los jóvenes 
cuya red permaneció estable entre los dos tiempos de evaluación se reparten entre las trayectorias constructivas y las vulnerabilizantes. Finalmente, la inmensa mayoría de los jóvenes cuya red se deterioró entre evaluaciones se sitúan en itinerancias vulnerabilizantes al final de la intervención.

Con mayor precisión, las redes que mejoran están caracterizadas por el abandono de los amigos perjudiciales, un cambio vinculado a la pareja, la implicación positiva de la propia familia y la de la pareja, y la aparición de una red cuando el joven estaba completamente aislado. A la inversa, las redes que se degradan incluyen la presencia de nuevos (o antiguos) amigos perjudiciales, la aparición de una pareja fuertemente inhibitoria o la ruptura de una relación muy constructiva y socializante, el regreso a casa de un progenitor que no posee bastantes habilidades paternas y perjudica el funcionamiento del joven o de la joven, o también la pérdida de soportes familiares como la familia que no quiere que el joven vuelva a casa después de su salida y, en definitiva, el aislamiento debido a la pérdida de apoyos.

En el caso de los jóvenes del PQJ, el desplazamiento hacia una red que ofrece apoyo, o menos inhibitoria, es generalmente debido a una elección basada en la reflexión. En diferentes casos, la acción del PQJ contribuyó a desarrollar las capacidades reflexivas de los jóvenes en cuanto a sus relaciones y las repercusiones sobre su trayectoria. La modificación comienza entonces y los profesionales intervinientes están presentes para echar una mano a los jóvenes en esta tarea compleja y a veces desgarradora de modificar su ambiente, incluso aunque suponga mejorar.

No obstante, la acción del PQJ es insuficiente para actuar de forma aislada en la trayectoria de los jóvenes, y la presencia de otros apoyos es indispensable. En este contexto, el apoyo de personas de referencia es muy necesario para facilitar el proceso de ruptura al final del proyecto. La intervención efectuada sobre la red de jóvenes debería por lo tanto apoyarse en la consolidación de los lazos con las personas de referencia, más que sobre la necesidad de abandonar a las personas inhibidoras, siendo la diversidad de la red un factor primordial.

La profundidad de los cambios en las redes, permitiendo generar modificaciones duraderas, supone que la continuidad de la relación de ayuda post-mayoría de edad constituye un factor de éxito y facilita, entre otros aspectos, la inserción social. Aunque el soporte institucional sea necesario cuando los jóvenes reciben servicios regulares de los centros de juventud, no parece suficiente para insertar a los jóvenes en un proyecto de vida constructivo si no continua más allá del periodo de protección institucional, de forma que pueda apoyar la experimentación social que sigue a ese periodo.

\section{Síntesis y discusión}

En definitiva, se debe priorizar en el proceso de selección a ciertos jóvenes de perfil severo, porque se benefician más con el programa. El camino que recorrieron es mayor que el de los jóvenes con dificultades menos severas. En efecto, si los pocos jóvenes que tienen recursos personales y sociales más importantes puedan beneficiarse de modo tangible del PQJ, su trayectoria muestra que la intervención ha influido sobre todo en una rápida movilización del joven en torno a un proyecto de cualificación. Esta movilización no requería de una intervención intensiva y de larga duración como la del PQJ, sobre todo porque estos jóvenes disponían ya en su red de personas que los apoyaban.

En este sentido, los resultados también mostraron que el compromiso del joven con el proceso de intervención era lo esencial para que se produjera su movilización. En efecto, hay que continuar identificando 
al PQJ como un recurso voluntario, verificando la motivación de los jóvenes hacia él. Desde el momento en que el joven se aleja y se sitúa de forma independiente a la intervención desde el PQJ, los resultados obtenidos son más moderados. Hay que encontrar entonces un medio para "atraer" a los jóvenes y después realizar una intervención intensiva a nivel de red, con el fin de alejarlo, por supuesto si esto es posible, de los principales inhibidores, pero sobre todo para intentar introducir allí nuevos referentes. Acentuar la intervención sobre la red supone también favorecer la inserción en actividades, en grupos comunitarios, donde tendrán la posibilidad de hacer nuevos encuentros y modificar progresivamente su red de contactos.

Además, la intervención en el marco del PQJ cuenta con el hecho de que uno de los elementos esenciales en la transición a la vida adulta del joven es la creación de una relación estable con un adulto. En esta perspectiva, el PQJ ha construido su dispositivo de intervención sobre todo en torno a la dimensión individual del joven. No obstante, nos consta que el apoyo de los pares es también importante. La intervención de grupo podría permitir desarrollar dinámicas interpersonales de apoyo y ayuda mutua entre los jóvenes, posibilitándoles compartir su experiencia individual. Contando con esa ayuda mutua, la intervención del grupo puede eventualmente desembocar en acciones sociales para actuar sobre ciertos bloqueos estructurales. Desde el punto de vista del análisis de datos de la evaluación del PQJ, resulta claro que esta estrategia de intervención sobre el grupo es un elemento importante, ya que permite transformar los juegos de poder de la relación individual profesional interviniente/joven, favoreciendo una ayuda mutua que se apoya en la experiencia entre los pares. Tales experiencias tendrán lugar en el marco del proyecto Apoyo a la vida autónoma (Proyecto SAVA, 2007-2011), desarrollando con los profesionales intervinientes y los jóvenes herramientas de intervenciones adaptadas a la complejidad de las transiciones a la vida autónoma.

\section{Conclusiones}

De manera general, el proyecto-piloto del PQJ condujo al desarrollo experto sobre las intervenciones a promover para preparar a los jóvenes que recibieron servicios de los centros de juventud en la vida autónoma, y apoyar su inserción social a la edad adulta. Cubre así un vacío relativo en este ámbito en Quebec (Goyette, 2006a). Este desarrollo experto y el carácter innovador del PQJ son reconocidos ahora, más allá de la red misma de los centros de juventud (Reid et Dubbing, 2006). Por otra parte, el Gobierno de Quebec confirmó la financiación para la generalización gradual del PQJ en el conjunto de los centros de juventud de Quebec, en el marco de la implantación de la Estrategia de acción juventud 2006-2009, y ha sido confirmada más tarde en la oferta de servicios de los centros de juventud.

Asimismo, más allá de la generalización del PQJ, los resultados de la evaluación testimonian la necesidad de proseguir con los esfuerzos, con el objetivo de responder mejor a las importantes necesidades del conjunto de los jóvenes que dejan un medio sustituto, apoyando su salida de los centros de juventud. En efecto, si el PQJ ofrecerá un apoyo adecuado a cerca de 500 jóvenes en todo Quebec, son más de 5.000 los jóvenes que dejan los centros de juventud para transitar a la vida adulta cada año. Estos jóvenes tienen recursos personales y sociales más importantes que los jóvenes del PQJ y probablemente no necesitan un apoyo intensivo y de larga duración como el PQJ. En cambio, tratándose de jóvenes que conocieron acogimientos familiares de larga duración, una intervención que tome 
más en consideración las dificultades y los juegos del paso a la vida adulta permitiría prepararlos mejor. Sobre este tema, nuestros trabajos reúnen las conclusiones de los informes, opiniones y algunos trabajos científicos quebequenses (Yergeau, 2007; Goyette et al., 2006a; Lanctot, 2006; Pauzé et al., 2004). En esta perspectiva, se impone el desarrollo de intervenciones referidas al apoyo de la salida de los centros de juventud del conjunto de los jóvenes protegidos. Podemos pensar en el desarrollo de herramientas que permitirían a los profesionales intervinientes de los centros de juventud delimitar mejor estas dimensiones.

A este respecto, sería particularmente interesante que sea analizada la situación del conjunto de jóvenes que reciben servicios de los centros de juventud cuando alcanzan los 16 años, con el fin de elaborar un plan adaptado para la preparación y para el tránsito a la vida adulta autónoma. Esta es la preocupación de los trabajos del ACJQ, a través de su comité Plan de progreso hacia la autonomía. En el núcleo de este enfoque, los compañeros de juventud quieren desarrollar una perspectiva y elementos comunes. Además de inscribirse directamente en las modificaciones aportadas a la Ley sobre protección de la juventud, buscando asegurar una mayor continuidad de los servicios, tal medida se inscribiría directamente en las medidas del Gobierno de Quebec para asegurar la concertación y la continuidad de las intervenciones con los jóvenes en dificultad.

Sobre el mismo tema, a pesar del entusiasmo reciente por la preparación para la vida adulta de los jóvenes protegidos, hay que señalar los pocos trabajos de reflexión existentes sobre su futuro. Así, Quebec no sabe verdaderamente lo que le ocurre a los jóvenes que protege porque no hay un estudio longitudinal sobre el futuro de los jóvenes que ha recibido servicios de los centros de juventud. Considerando el giro orientado a la gestión de resultados inicia- do por el Gobierno de Quebec, y el hecho de que la red de los centros de juventud otorga sumas anualmente de más de $950 \mathrm{Ms}$, ciertamente la puesta en marcha de este estudio es prioritaria.

\section{Referencias bibliográficas}

ACJQ (2006): Guide d'application du programme Qualification des Jeunes. Montreal.

Bidart, C. (2006): Devenir adulte aujourd'hui: perspectives internationales: París, INJEP/L'Harmattan.

Bidart, C. y J. Charbonneau (2007): The Contextual Name Generator: A Good Tool for the Study of Sociability and Socialization. Communication présentée au XXVIIe Congrès de l'International Network for Social Network Analysis, Corfou, Grèce, mai 2007, Inédits 2007-06, INRSUrbanisation, Culture et Société, 35 p.

Bellot, C.; Goyette, M. (2006): "S'insérer. Un enjeu majeur pour les jeunes en difficulté". Intervention, 125, pp. 17-26.

Charbonneau, J. (2003): Adolescentes et mères: histoires de maternité précoce et soutien du réseau social. Ste-Foy, PUL

Daining, C. y DePanfilis, D. (2007): "Resilience of youth in the transition from out-of-home care to adulthood". Children and Youth Services Review, 29, pp. 1158-1178.

Dumaret, A.-C.; Coppel-Batsch, M. y Couraud, S. (1997): "Adult Outcome of Children Reared for Long-term Periods in Foster Families". Child Abuse \& Neglect, 21 (10), pp. 911-927.

Frechon, I. (2005): Les stratégies féminines d'entrée dans la vie adulte. In E. Callu; J.-P. Jurmand; A. Vulbeau (Eds.): La place des jeunes dans la cité. Tome 2, Espaces de rue, espaces de parole (pp. 215-232). París, L'Harmattan.

Goyette, M. (2006a): "L'insertion socioprofessionnelle et la préparation à la vie autonome des jeunes pris en charge par l'État au Québec : Vers quelles interventions?". Sociétés et de la juventuds en difficulté. Revue en ligne.

Goyette, M.; Bellot, C. y Panet-Raymond, J. (2006a): Le projet Solidarité De la juventud. Dynamiques 
partenariales et insertion des jeunes en difficulté. Québec, PUQ, Coll. PSIS.

Goyette, M. (2007a): Promoting autonomous functionning among youth in care: A program evaluation. In V. R. Mann-Feder (Ed.), Transition or eviction: Youth exiting care for independent living (pp. 89-105). San Francisco, Wiley Subscription Services.

Goyette, M. (2007b): "Réseaux sociaux et insertion socioprofessionnelle des jeunes en difficulté". Intervention (127), pp. 48-57.

Goyette, M.; Chénier, G.; Noël, V.; Poirier, C.; Royer, M.-N. y Lyrette, É. (2006b): Comment faciliter le passage à la vie adulte des jeunes en centre de la juventud. Évaluation de l'intervention réalisée du projet d'intervention intensive en vue de préparer le passage à la vie autonome et d'assurer la qualification des jeunes des centres jeunesse Québec. Montréal, Rapport de recherche remis à l'ACJQ et au CNPC.

Goyette, M.; Royer, M.-N.; Noël, V. y Chénier, G. (2007a): Projet d'intervention intensive en vue de préparer le passage à la vie autonome et d'assurer la qualification des jeunes des centres jeunesse Québec. Rapport final d'évaluation. Montréal, Remis au CNPC et à l'ACJQ.

Goyette, M. y Turcotte, D. (2004): "La transition vers la vie adulte des jeunes qui ont vécu un placement: un défi pour les organismes de protection de la jeunesse". Service social, 51 (1), pp. 30-44.

Jahnukaiken, M. (2007): "Hi-risk youth transitions to adulthood: A longitudinal view of youth leaving the residential education in Finland". Children and Youth Services Review, 29, pp. $637-654$.

Kufedlt, K. (2003): "Graduates of Guardianship Care: Outcomes in Early Adulthood". In Child Welfare: Connecting Research, Policy and Practice (pp. 203-216). Wilfred Laurier University Press.

Lanctôt, N. (2006): "Les adolescentes prises en charge par le centre de la juventud: Que deviennent-elles au tournant de la vingtaine?". Défi de la jeunesse, 12 (2), pp. 3-7.

Manseau (1997): Les grossesses chez les adolescentes en internat: Le syndrome de la conception immaculée. Thèse de doctorat. Université du Québec à Montréal, Montréal.

Maunaye, E. y Molgat, M. (2003): Conclusion. In E. Maunaye, M. Molgat (eds.): Les jeunes adultes et leurs parents. Autonomie, liens familiaux et modes de vie (pp. 223-226). Sainte-Foy, PUL et l'IQRC.

Morin, A. (2003): Projet d'intervention intensive en vue de préparer le passage à la vie autonome et d'assurer la qualification des jeunes des centres jeunesse Québec - Bilan de l'an 1. Montréal, ACJQ.

Morin, A. (2004): Projet d'intervention intensive en vue de préparer le passage à la vie autonome et d'assurer la qualification des jeunes des centres jeunesse Québec. Bilan de l'an II. Montréal, ACJQ.

Pauzé, R.; Toupin, J.; Déry, M.; Mercier, H.; Joly, J. y Cyr, M., et al. (2004): Portrait des jeunes âgés de o à 17 ans référés à la prise en charge des centres jeunesse Québec, leur parcours dans les services et leur évolution dans le temps (Section 6: Portrait des jeunes devenus adultes). Sherbrooke, GRISE.

Pecora, P. J.; Kessler, R. C. y O'Brien, K., et al. (2006): "Educational and employment outcomes of adults formerly placed in foster care". Children and Youth services Review, 28 (12), pp. 1459-1481.

Reid, C. (2007): The transition from state care to adulthood: International examples of best practices. Dans V. R. Mann-Feder (ed.): Youth leaving care: How do they fare? (pp. 33-49). San Francisco, Wiley Subscription Services.

Reid, C. y Duddling, P. (2006): Building a Future Together: Issues and Outcomes for TransitionAged Youth. Ottawa, CECW. CWLC.

Rutman, D.; Hubberstey, C.; Feduniw, A. y Brown, E. (2006): When Youth Age Out of Care Bulletin of Time 2 Findings. Victoria, Research Initiatives for Social Change unit, School of Social Work, University of Victoria.

Tweddle, A. (2007): Youth leaving care: How do they fare? In V. R. Mann-Feder (ed.): Transition or eviction: Youth exiting care for independent living (pp. 15-31). San Francisco, Wiley Subscription Services.

Yergeau, E.; Pauzé, R. y Toupin, J. (2007): “L'insertion professionnelle et l'adaptation psychosociale des jeunes adultes ayant reçu des services des centres de la juventud". Intervention (127), pp. 58-69. 


\section{Notas}

${ }^{1}$ Las investigaciones reflejadas en este artículo han sido realizadas gracias a la financiación y el apoyo de la «Stratégie nationale pour la prévention du crime», del gobierno de Canadá, en colaboración con el «Ministère de Sécurité Publique» de la región de Québec, y el "Institut des Services et des Politiques de Santé des Instituts de Recherche en Santé» de Canada. El informe final: Goyette, M., Royer, M.-N., Noël, V., et Chénier, G. (2007a): Projet d'intervention intensive en vue de préparer le passage à la vie autonome et d'assurer la qualification des jeunes des centres jeunesse Québec. Rapport final d'évaluation. Montreal, CNPC et ACJQ. (ISPS-IRSC 2009).

${ }^{2}$ Los centros de juventud son establecimientos que ofrecen servicios especializados a los jóvenes en su mismo territorio. Han sido creados gracias a una reagrupación de servicios a la infancia (los centros originarios de protección de menores y de jóvenes, algunos años antes centros de servicios sociales, y los servicios y centros de readaptación para los jóvenes en dificultad). Se trata de crear así, bajo la misma organización, una atención integral en protección de la juventud y readaptación psicosocial. Los centros de juventud tienen como mandato prioritario la aplicación de la Ley sobre protección de la juventud, la Ley sobre el sistema de justicia penal para adolescentes y la Ley sobre los servicios sociales y de salud. Los dieciséis centros de juventud de Quebec dan servicios a unos 100.000 niños, jóvenes y familias en dificultad cada año.

${ }^{3}$ Para una descripción más detallada del PQJ ver Morin (2004; 2003) y ACJQ (2006).

${ }^{4}$ La visión subjetiva del profesional interviniente fue objetivada por un cuadro de referencia. Esta visión fue luego confrontada con la visión externa de la coordinadora provincial, con el objetivo de validar la elección del joven. Los cuadros de severidad y de disposiciones personales han sido desarrollados a partir del cuadro de referencia indicado (véase apartado 3.1).
${ }^{5}$ Estas escalas, aunque no fueron objeto de un proceso de validación estadística completa, demostraron sin embargo una validez suficiente para ser utilizadas tal y como se diseñaron, es decir, a título indicativo de la evolución del perfil de los jóvenes a través del proceso de intervención. En efecto, la consistencia interna de ambas escalas, medidas por el alfa de Cronbach (?), es muy próxima o superior a o,7. En T6 de medida, la escala de severidad del perfil tiene un alfa de o,6987 y la de las disposiciones personales es aún más consistente, con un alfa de o,8661. Los diferentes ítems de las escalas están fuertemente correlacionados entre ellos y atestiguan así que miden el mismo fenómeno, en un caso la severidad del perfil de los jóvenes, en otra la de sus disposiciones personales.

${ }^{6}$ La cuestión de la repercusión de las relaciones sociales en el proceso de inserción de los jóvenes ha sido tratada en el № 127 de la revista Intervención. (Goyette, 2007b).

DiRECCIÓN DEL AUTOR: Martin Goyette. Escuela Nacional de Administración Pública. Universidad de Quebec.

Correo electrónico: martin.goyette@enap.ca.

Fecha de recepción del artículo: 15.X.2009

Fecha de aceptación definitiva: 18.I.2010

COMO CITAR ESTE ARTÍCULO:

Goyette, M. (2010): "El tránsito a la vida adulta de los jóvenes atendidos desde los servicios sociales". Pedagogía Social. Revista Interuniversitaria, 17, pp. 43-56. 